\title{
PREJUICIOS LINGÜÍSTICOS DE LA JUVENTUD CUBANA HACIA EL INGLÉS
}

\author{
Daniel Pinto Pajares
}

\section{(c) $(7) \Theta$}

Doi: https://doi.org/10.15517/rfl.v44i2.34697

URL: https://revistas.ucr.ac.cr/index.php/filyling 



\title{
PREJUICIOS LINGÜÍSTICOS DE LA JUVENTUD CUBANA HACIA EL INGLÉS
}

\section{LANGUAGE PREJUDICES OF CUBAN YOUTH TOWARDS ENGLISH}

\section{Daniel Pinto Pajares}

\begin{abstract}
RESUMEN
El inglés es una de las lenguas que más valoraciones suscita en todo el mundo. Apreciaciones como lengua internacional o lengua de comunicación y sus supuestas cualidades intrínsecas han generado una serie de preconceptos que no se sostienen desde un punto de vista lingüístico. Sin embargo, se trata de prejuicios que están latentes y que configuran un espacio hegemónico en el que el inglés ocupa un rol subordinante. Esta investigación analiza los prejuicios lingüísticos hacia el inglés que presenta una muestra de informantes cubanos comprendidos entre los 18 y los 30 años. Así, se toma como objeto de este estudio sociolingüístico a la juventud cubana en tanto que agente social que marcará el rumbo de Cuba en las próximas décadas. El trabajo de campo se ha realizado en La Habana y se ha empleado una metodología cualitativa basada en entrevistas semi-dirigidas como herramienta para extraer los datos.

Palabras clave: Prejuicio lingüístico, Cuba, inglés, lengua internacional, heterogeneidad lingüística.
\end{abstract}

\begin{abstract}
English is one of the most valuated language around the world. Valuations as global language or language of communication and its intrinsic supposed qualities to the language have organized a string of preconceptions that are not held from a linguistic point of view. However, they are latent prejudices which form a hegemonic space where English takes a principal role up. This research analyses the linguistic prejudices towards English presented by a sample of Cuban informants between 18 and 30 years old. Thus, the Cuban youth, as the social agent that is going to mark the course of Cuba in the coming decades, is the object of this sociolinguistic research. The fieldwork was conducted in Havana and has a qualitative methodology that utilizes a semi-structured interview as the data collection tool.
\end{abstract}

Key words: Linguistic prejudice, Cuba, English, global language, linguistic heterogeneity.

Dr. Daniel Pinto Pajares. Universidade de Vigo. Doctor por la Universidade de Vigo e investigador del grupo Gramática, Discurso e Sociedade (GRADES). España.

Correo electrónico: danielpinto_92@hotmail.com

Recepción: 16- 05- 2018

Aceptación: 19- 06- 2018 


\section{Introducción}

Las opiniones en torno a las lenguas del mundo han sido una constante a lo largo de los siglos. Valoraciones jerárquicas de diversa índole catalogan las lenguas mediante parámetros extralingüísticos. Sapir (1921, p. 234) afirmaba que "all attempts to connect particular types of linguistic morphology with certain correlated stages of cultural development are vain". Apreciaciones sobre la supuesta facilidad o dificultad de las lenguas, sobre la excelencia literaria de las mismas o sobre el nivel de desarrollo que comportan, entre otras, extrapolan cuestiones estrictamente gramaticales al terreno sociocultural.

En este trabajo se analizan los prejuicios lingüísticos que presenta la juventud cubana hacia el inglés. Ante el nuevo marco político y económico que se está abriendo en Cuba, resulta interesante conocer las opiniones de un grupo demográfico que dentro de poco tiempo será el encargado de marcar el rumbo del país. Asimismo, el hecho de elegir el inglés como lengua objeto de prejuicios lingüísticos se justifica atendiendo a la cercanía geográfica entre Cuba y EE.UU., un país anglófono, y al restablecimiento de relaciones entre ambos países, lo cual permitirá presumiblemente la entrada del inglés en Cuba como un elemento simbólico de cercanía y cordialidad.

\section{Hipótesis}

Esta investigación se sustenta en la idea de que la juventud cubana incorpora en su acervo ideológico diversos prejuicios lingüísticos hacia el inglés. Concretamente, los prejuicios que se exponen a continuación son las hipótesis de las que parte este trabajo:

1. El inglés es la lengua internacional por excelencia debido a su presencia global.

2. El inglés permite la comunicación entre hablantes que no comparten la misma lengua.

3. Entre todas las variedades lingüísticas del inglés, la de EE.UU. es la más correcta.

4. El inglés que aprende una persona cubana como L2 es de peor calidad que el de un hablante nativo.

\section{Marco teórico}

Entre otros aspectos, la sociolingüística investiga las opiniones que la sociedad emite sobre las lenguas como juicios de valor que, sin embargo, contribuyen a organizar argumentaciones falaces sobre la base de supuestos rasgos inferiores y superiores de unas y otras lenguas.

Iglesias (2012, p. 6) señala que los prejuicios son tipos de actitudes en las que todos sus componentes se manifiestan en sentido negativo. El componente cognitivo genera estereotipos negativos, el componente afectivo produce sentimientos de rechazo y el componente conativo aviva la tendencia a discriminar. Sin embargo, otros autores como Allport (1962, p. 21) reconocen que los prejuicios pueden ser tanto a favor como en contra de un fenómeno social, por lo que la actitud puede ser favorable o desfavorable, pero siempre vinculada a una creencia generalizada. En el caso que nos ocupa, se detecta y evalúa una serie de prejuicios fundamentados en creencias positivas hacia el inglés.

Nuestro lenguaje teórico analiza y describe las opiniones mediante un formato proposicional en cuya base se encuentran creencias que no pueden ser descompuestas y que, a su vez, forman sistemas de creencias más elaborados (Van Dijk, 1998, p. 22). En tanto que 
estructuras mentales, las creencias se basan en generalizaciones, lo cual supone una inmediata simplificación, pero el conocimiento humano necesita de esas simplificaciones para ordenar y entender el mundo. Esta racionalización de la realidad se afirma con base en consideraciones que cumplen una función irracional para quien la sustenta (Ackerman y Jahoda, 1950, p. 4).

Este proceso se denomina categorización y equivale a producir generalizaciones que constituyen la base de los prejuicios. Consecuentemente, los prejuicios son predisposiciones inmotivadas en la medida en que no se fundamentan en hechos suficientes o demostrables objetivamente. Generalmente, el individuo admite una categoría solo si esta le reafirma en sus creencias previas (Allport, 1962, p. 39). Por lo tanto, la categorización conduce al individuo a reafirmar su modo de vida, lo cual le conduce a entrar en el prejuicio. El sujeto sobrestima aquello que concuerda con la idea que se había formado previamente de las cosas y rechaza aquello que no encaja en su modelo preestablecido. Así, se identifica con un endogrupo antes de formarse la idea de un exogrupo, pues este supone una amenaza para él. Pero Spinoza (2000, p. 184) recuerda que las personas categorizan las cosas de la naturaleza en virtud de prejuicios, pues nada que no haya sido obra humana puede ser perfecto o imperfecto en tanto que la naturaleza no trabaja en torno a un fin.

Concebir los prejuicios en términos de creencias no los reducen exclusivamente al terreno mental. Los prejuicios son formas de conocimiento colectivas, en el sentido de que no se pueden reducir a intenciones individuales, porque se adquieren socialmente (Kroskrity, 2010, p. 200). Teniendo en cuenta que las lenguas son los instrumentos básicos para la socialización, existe un amplio repertorio de prejuicios sobre las lenguas.

Los prejuicios lingüísticos inciden directamente en los hablantes, generando ideologías discriminatorias hacia comunidades lingüísticas debido a que hablan lenguas que, a pesar de haberse adquirido espontáneamente, no gozan de prestigio social. Precisamente, las comunidades más expuestas a los prejuicios lingüísticos y, por extensión, a los prejuicios culturales, son a menudo las que hablan lenguas minorizadas que no cuentan con ninguno o muy escaso respaldo institucional y que les cuesta resistir el avance del discurso hegemónico. Un discurso que eleva a la condición de superioridad a aquellas lenguas que tienen tras de sí grandes imperios culturales y económicos mientras que desprecia la diversidad lingüística, condenando al ostracismo y al menosprecio a aquellas lenguas que, desde una perspectiva occidental y capitalista, no producen beneficios económicos relevantes.

En este trabajo se analiza este tipo de valoraciones hacia la lengua inglesa por parte de la juventud cubana. Las opiniones que se vierten a menudo sobre el inglés hacen que se configure como una lengua con ciertas propiedades innatas que nada tienen que ver con lo lingüístico, y esta investigación explora la presencia o ausencia de dichas calificaciones en un grupo poblacional muy concreto de la sociedad cubana.

\section{Antecedentes}

Si bien la sociolingüística cubana ha abordado los prejuicios lingüísticos presentes en la sociedad de Cuba, estos trabajos se centran por lo general en las valoraciones hacia el castellano, ya sea hacia las variantes cubanas o hacia el resto de modalidades de los países hispanohablantes. Sin embargo, hasta el momento hay un vacío de investigaciones sobre los prejuicios lingüísticos hacia el inglés en Cuba. A continuación se indican estudios realizados en Cuba con propósitos similares a los nuestros que, en algunos casos, abordan percepciones lingüísticas tales como los prejuicios y, en otros, aspectos actitudinales. 
Gregori Torada (1993-1994) ofrece un estudio que amplía las fronteras del marco cubano para abordar los prejuicios lingüísticos que sufren las distintas variedades del castellano caribeño. Uno de los resultados más destacables revela la minusvaloración de la que es objeto el castellano de Cuba por parte de los propios cubanos. La autora advierte de los peligros de esta discriminación lingüística y propone ciertas medidas para encarar estas valoraciones negativas.

Un estudio actitudinal diacrónico muy relevante es el de Canals Fleitas (1999), donde se explora el desarrollo histórico, desde 1791 hasta 1999, de las actitudes lingüísticas del habanero hacia el habla de sujetos marginados a lo largo de esos siglos, como el negro, el homosexual o los practicantes de rituales religiosos africanos. La autora cuestiona la influencia de la política lingüística en el declive de actitudes negativas durante tres etapas: la colonial, la pseudorepublicana y la revolucionaria.

Alfaraz (2002) analiza las percepciones de un grupo de cubanos residentes en Miami hacia las distintas variedades del castellano y hacia el castellano anterior y posterior a la Revolución Cubana. Las percepciones más favorables se detectan en las variedades del castellano procedente de países con un nivel económico alto y en el castellano prerrevolucionario. La autora subraya un componente racial en las percepciones negativas hacia el castellano habanero posterior a la Revolución en tanto que sufrió una africanización como consecuencia del aumento de la población negra en La Habana durante ese periodo.

La investigación de Watson (2006) es una de las que más ha contribuido al estudio actitudinal de la sociedad cubana. En este caso, se analiza las actitudes lingüísticas de cubanoestadounidenses y habaneros blancos con un nivel educativo alto hacia las distintas variedades del castellano. La investigación determina que los cambios políticos instaurados en Cuba a partir de 1959 alteraron el léxico coloquial de personas con un nivel educativo alto. Asimismo, se investigan algunos trazos lingüísticos altamente estigmatizados, como la geminación y la lateralización.

Por su parte, Fuster (2012) analiza las percepciones y actitudes lingüísticas de 72 habaneros y santiagueros para detectar si ciertas variables sociales son determinantes en los resultados. La autora descubre que el origen y el estatus social influyen decisivamente en las percepciones y actitudes de las personas cuestionadas. Dado que históricamente los habitantes de Santiago de Cuba han sufrido ciertos prejuicios culturales por parte de sus compatriotas, su variedad del castellano es igualmente la más estigmatizada. Así, la autora detecta motivos étnicos y socioeconómicos en la base de los prejuicios lingüísticos.

Como parte del Proyecto LIAS (Linguistic Identity and Attitudes in Spanishspeaking Latin America), Sobrino Triana, Montero Bernal y Menéndez Pryce (2014) analizan pormenorizadamente las actitudes y los prejuicios lingüísticos de una muestra de 400 personas cubanas hacia su propia variedad del castellano y las de otros hispanohablantes. La investigación aborda las denominaciones, las percepciones cognitivo-linguísticas y las actitudes afectivas hacia el castellano. Los resultados revelan actitudes más positivas que los que descubrieron algunos trabajos previos. El castellano habanero aparece como la variedad más prestigiosa, argumento sostenido por elementos de carácter extralingüístico, mientras que una minoría de los cuestionados defiende la idea de la igualdad de las lenguas.

Asimismo, se perciben algunos prejuicios negativos destacables. En consonancia con estudios anteriores, la variedad oriental del castellano de Cuba es la más estigmatizada debido a criterios lingüísticos, como una supuesta mala pronunciación, y a criterios extralingüísticos, 
como el nivel cultural de sus hablantes. Pese a que la variedad habanera aparezca como la más prestigiosa, algunos informantes detectan chabacanería léxica en el uso de jerga como asere o qué volá.

Respecto a los prejuicios generales del castellano, la investigación revela la predilección de las personas entrevistadas por la corrección gramatical, asociándola en buena medida con las normas de la RAE y el castellano de España. Además, este último se toma como modelo de unidad lingüística para una proporción considerable de los informantes.

Estos criterios de corrección e incorrección se abordan en Velázquez Pratts (2009), donde se toma una muestra de 120 participantes de la ciudad cubana de Holguín para tratar sus apreciaciones respecto a las variantes nacionales y de otros países hispanohablantes.

Respecto a investigaciones sociolingüísticas que aborden los prejuicios lingüísticos en la juventud cubana, existe un vacío importante que este trabajo pretende cubrir. No obstante, destacan las investigaciones de Dohotaru (1998-1999; 2000; 2002), que trata ciertos rasgos lingüísticos del habla coloquial de universitarios cubanos, tales como la variación de los fonemas $/ \mathrm{s} /, / \mathrm{R} / \mathrm{y} / \mathrm{l} /$.

Los trabajos anteriores se perfilan como los antecedentes más importantes para esta investigación. Cada uno de ellos demuestra las diferentes perspectivas desde las que se ha trabajado en Cuba el tema que tratamos.

\section{Metodología}

Para abordar un tema de estas características, es preciso acercarse a la metodología llevada a cabo para extraer los datos que posteriormente se analizan. Tanto la herramienta de trabajo como la muestra de informantes son las mismas que se han utilizado en un trabajo precedente que pone la atención en las ideologías lingüísticas de la juventud cubana hacia el inglés (Pinto Pajares, 2016). Así pues, para aprovechar la estancia en La Habana entre julio y agosto de 2016, se consideró oportuno recabar datos para sendas investigaciones a partir de las mismas personas entrevistadas.

\subsection{Muestra de informantes}

A continuación detallamos los criterios seguidos para seleccionar a las personas entrevistadas de este estudio:

1. Número de informantes: se ha entrevistado a un total de 10 personas. El límite de tiempo de la estancia en La Habana condicionó en cierto modo el número de personas a las que se accedió. Sin embargo, la causa principal de esta cantidad reside en la propia herramienta de extracción de datos. En tanto que estudio cualitativo, no se busca generar estadísticas para representar a un grupo demográfico en su totalidad, sino aproximarse a las tendencias ideológicas que se manifiesten.

2. Paridad de sexos: se ha seleccionado a 5 hombres y a 5 mujeres.

3. Edad: la franja de edad de las personas entrevistadas oscila entre los 18 y los 30 años. En tanto que se investiga a la juventud, es necesario especificar qué se entiende en este estudio por juventud, ya que los criterios pueden ser múltiples. En este caso, se ha descartado a menores de edad por cuestiones legales y a mayores de 30 años, ya que una amplitud del tramo etario generaría resultados más dispares. Asimismo, son 
precisamente las personas que se encuentran en esta franja de edad quienes marcarán el rumbo político, económico y social de Cuba en un futuro próximo.

4. Origen geográfico: se ha privilegiado la diversidad frente a la uniformidad respecto al origen de los entrevistados.

5. Formación académica: nuevamente, se ha garantizado la variedad en cuanto al nivel de instrucción de la muestra de las personas entrevistadas.

En la siguiente tabla se detallan los rasgos individuales de cada persona entrevistada:

Cuadro 1. Perfil de las personas entrevistadas

\begin{tabular}{|c|c|c|c|c|}
\hline Pseudónimo & Sexo & Edad & Origen & Nivel de estudios \\
\hline Natalia & M & 18 & Pinar del Río & Preuniversitario \\
\hline Roberto & $\mathrm{H}$ & 19 & Guanabacoa (La Habana) & Carrera universitaria \\
\hline Carla & M & 21 & Camagüey & Carrera universitaria \\
\hline Emma & M & 22 & La Habana & Carrera universitaria \\
\hline Lorena & M & 23 & Matanzas & Carrera universitaria \\
\hline Yoel & $\mathrm{H}$ & 25 & Bayamo (Granma) & Educación Técnica Profesional \\
\hline Diana & M & 26 & Santiago de Cuba & Carrera universitaria \\
\hline Lucas & $\mathrm{H}$ & 27 & Holguín & Preuniversitario \\
\hline Alejandro & $\mathrm{H}$ & 28 & La Habana & Educación Técnica Profesional \\
\hline Mario & $\mathrm{H}$ & 30 & Cienfuegos & Secundaria \\
\hline
\end{tabular}

Como se observa, no aparecen los nombres reales de las personas entrevistadas, sino que se les ha sustituido por pseudónimos para asegurar su anonimato y privacidad. Por otro lado, en el origen geográfico se indica entre paréntesis la provincia de Cuba a la que pertenece la ciudad que la acompaña, siempre y cuando no coincida el nombre de la ciudad con el de su correspondiente provincia.

\subsection{Trabajo de campo}

En la investigación sociolingüística, la metodología cualitativa domina desde mediados de la década de 1990 ya que la mayoría de los aspectos linguiísticos están determinados por 
factores sociales, culturales y situacionales (Dörnyei, 2007, p. 36). Dado que el significado se construye socialmente, es necesario enfocarse en el discurso de los individuos a través de una conversación que garantice la exteriorización de estructuras ideológicas profundas. En consecuencia, esta investigación utiliza una metodología cualitativa en tanto que el objetivo se centra en entender lo particular y distintivo, sin necesidad de generalizar al conjunto de la sociedad ni a otros contextos. A continuación se detallan las tres fases que conforman la metodología de este trabajo.

\subsubsection{Cuestionario}

El acceso a personas que colaborasen con esta investigación no ha contado con obstáculos reseñables dada la buena predisposición de la juventud cubana. Se han buscado informantes en lugares frecuentados mayoritariamente por personas jóvenes, como escuelas de Secundaria, la Universidad de La Habana y centros de trabajo. Hemos encontrado un grupo numeroso de posibles entrevistados que, pese a ser un número alto de personas, presentaban perfiles similares. Por consiguiente, a partir de los cuestionarios rellenados por todos ellos, se han priorizado algunos requisitos como la variedad geográfica, dado que una gran parte de estos jóvenes eran oriundos de La Habana. En dicho cuestionario se han incluido datos como la edad, el origen geográfico, el nivel de estudios y otras informaciones sin relevancia investigadora pero metodológicamente importantes para que se familiarizasen con el tema que se iba a tratar posteriormente y así aliviar posibles tensiones.

\subsubsection{Entrevista cualitativa}

Para extraer datos relevantes para el análisis, la entrevista cualitativa o semidirigida ha sido la herramienta con la que se ha obtenido el discurso de los informantes. Este tipo de entrevista se caracteriza por permitir un carácter abierto en las preguntas, de manera que no se siga necesariamente un esquema prefijado de preguntas y secuencias (del Rincón Igea, 1995). Para Bourdieu (1997, p. 83), algunas herramientas de investigación como los cuestionarios solo pueden ofrecer resultados inverosímiles dado que, a menudo, los entrevistados no se han planteado nunca las preguntas que se les dirige.

Como se mencionó anteriormente, esta entrevista semidirigida se ha utilizado para investigar conjuntamente dos aspectos relacionados: los prejuicios lingüísticos y las ideologías lingüísticas de la juventud cubana hacia el inglés. El primer tema es el que se trata en esta ocasión mientras que el segundo se ha desarrollado en un trabajo previo. De esta manera, si bien existía un esquema para dividir las preguntas en bloques temáticos, los datos obtenidos para explorar los prejuicios lingüísticos no han contado con una estructuración previa de preguntas, sino que se extraen del discurso general de cada informante y de ciertas cuestiones concretas a lo largo de la entrevista.

La propia herramienta de trabajo ha permitido un estilo más conversacional y cercano, de forma que ciertas respuestas han aportado datos para tratar en ambos estudios. La entrevista cualitativa no puede ser un diálogo en sentido estricto porque no existe una relación igualitaria entre los interlocutores, puesto que uno busca el conocimiento y el otro sirve de medio para el interés del investigador (Kvale, 2006, p. 483). Más bien, se da un reparto asimétrico del poder que permite al investigador monopolizar la interpretación a partir de una conversación instrumental. Sin embargo, se ha creado un clima de cercanía que ha permitido 
que los informantes entendieran la entrevista como una conversación entre iguales, propiciando la aparición de numerosos datos, de tal manera que de una misma respuesta se ha obtenido información relevante para este trabajo y para el de las ideologías lingüísticas.

\subsubsection{Código ético}

Todas las personas entrevistadas han firmado un código ético donde se plasma el compromiso de confidencialidad y anonimato por parte del investigador. Los datos recogidos tienen una finalidad exclusivamente académica y las personas entrevistadas son libres de rechazar a posteriori su participación en el estudio.

\section{Análisis de los resultados}

\subsection{Caracterización del inglés}

A lo largo de la entrevista han aparecido distintos calificativos para el inglés a partir de los que se infiere una cierta visión del mundo de los informantes. Lo realmente destacable no es el epíteto asociado a la lengua inglesa sino el propio hecho de su aparición, dado que las preguntas no iban encaminadas a que aportasen este dato, sino que ha sido una iniciativa de todas las personas entrevistadas. Repartidas en diferentes puntos de la conversación, cada informante ha revelado una denominación particular del inglés.

El calificativo más repetido es el que confiere al inglés la cualidad de lengua universal, siendo declarado por la mitad de las personas entrevistadas. Cabe puntualizar que una de estas personas concretó que la variedad del inglés de Inglaterra es la más universal, mientras que el resto de estas personas entrevistadas otorgan dicha característica a la lengua inglesa en su conjunto, sin destacar en este caso unas variedades por encima de otras.

La segunda denominación más repetida es la de internacional, sostenida por tres informantes. Dentro de este grupo, Natalia no solo concede al inglés esta propiedad, sino que lo hace en grado superlativo: "en estos momentos, es la lengua más internacional que existe". De esta manera, unos informantes entienden la cualidad de internacional más bien como una esencia única y exclusiva del inglés mientras que Natalia la interpreta como una propiedad de la que disponen varias lenguas, siendo el inglés la que la posee en mayor medida.

Estos dos grupos de informantes dibujan un escenario de ámbito mundial en el que, supuestamente, el inglés tiene presencia en todos los rincones. No perciben esta lengua como ligada a una comunidad lingüística o territorio concretos, con sus correspondientes parámetros culturales e idiosincráticos, sino que la entienden como un fenómeno lingüístico único, capaz de romper fronteras y abarcar el conjunto del planeta. Las personas participantes no evalúan el origen de esa internacionalidad, aceptándola como algo lógico. Pennycook (1994, p. 255) cuestiona esta aceptación del inglés internacional como si fuera parte de un orden natural desmarcado de las fuerzas políticas y económicas del capitalismo que lo ha impuesto. Para justificar la concepción de internacional, no todos los entrevistados aportan razones, pero quienes lo hacen aluden al factor comunicativo.

En este sentido, Diana sí argumenta su defensa del inglés como lengua internacional alegando que lo es porque "muchos países lo utilizan para comunicarse cuando las personas no saben el habla materna del país en el que se encuentran”. Asimismo, Mario sostiene que el 
inglés es universal porque "cuando vas a viajar, y no solamente a países de habla inglesa, sino a otros que no hablen inglés como Francia, uno se puede comunicar". Ambos informantes detectan en el inglés la propiedad de ser entendido en muchos países como justificación para darle una denominación tan abarcadora. Sobreentienden que todas las personas, independientemente de su nacionalidad y lengua materna, poseen unas nociones mínimas de inglés como para comunicarse, por lo que el inglés actuaría como lengua franca. Este término procede del árabe lisan-alfarang y su significado original se refería a una lengua intermedia utilizada por los árabes y los viajeros de Europa occidental cuando se encontraban (House, 2003, p. 557). Para Edwards (1994, p. 1), las lenguas francas "serve as aids to cross-groups understanding, and [...] usually represent the language of a potent and prestigious society". La introducción de esta idea se pasa por alto a menudo para desconectar dos características correlacionadas: la internacionalidad de una lengua y el poder político y económico de los Estados que fomentan dicha lengua. De las aseveraciones de los informantes anteriores se desprende la idea de que la internacionalidad del inglés es una propiedad tan consustancial a la lengua como lo puedan ser sus categorías gramaticales. Estos prejuicios lingüísticos son alentados por numerosas instituciones percibidas como legítimas y con capacidad de imponer ideas falaces que posteriormente son reproducidas por personas que no son lingüistas y que no tienen por qué serlo.

La responsabilidad principal de que estas ideas estén tan extendidas recae a menudo en algunos lingüistas que, con todos los recursos académicos a su alcance, contribuyen a la difusión de prejuicios que privilegian a las lenguas ya privilegiadas. En este sentido, House (2003, p. 557) asocia al inglés la propiedad de flexibilidad funcional como característica consustancial a la estructura interna de la lengua, lo cual la hace ser capaz de expandirse internacionalmente. Estudios de este tipo presentan el inglés como una lengua neutral "que debe ser aceptada como el modo más apropiado para llegar a un entendimiento común" (Moreno Cabrera, 2004, p. 61).

Este prejuicio lingüístico presenta diferentes grados de asimilación entre las personas entrevistadas. Si bien los informantes anteriores elaboran un razonamiento para sostener su tesis, otros, sin embargo, no construyen una línea argumental, sino que entienden la característica de internacionalidad o universalidad hacia el inglés como un axioma. De tal manera que, para estos entrevistados, el inglés goza de esta cualidad como una cuestión natural que no necesita deducirse a partir de postulados previos porque lo consideran una proposición evidente.

En un grado menor de intención universalizadora se sitúa Roberto, que afirma que el inglés "es uno de los idiomas más importantes del mundo". En este caso, la cualidad que le otorga al inglés es relacional, y no absoluta como en los casos anteriores. Mientras que los informantes a los que se hizo referencia anteriormente confieren al inglés una naturaleza categórica, Roberto señala una cualidad del inglés que lo es en relación con otras lenguas. En otras palabras, para el informante, el inglés es importante debido a una serie de criterios que cumple dicha lengua pero que también cumplen otras lenguas, mientras que los entrevistados anteriores asumen el inglés como lengua universal o internacional per se. Por consiguiente, de la declaración de Roberto se infiere una competición entre lenguas por alcanzar el grado de "importante". Los condicionantes para alcanzar tal categoría no son señalados en ese mismo instante por el informante, pero sí se dejan ver a lo largo de la entrevista. De esta manera, el inglés es importante porque sirve como recurso para "obtener trabajo, para aumento de sueldos". 
Por último, solamente una informante no ha señalado en ningún momento de la entrevista ningún calificativo hacia el inglés, infiriéndose así que lo pone al mismo nivel que el resto de lenguas en cuanto a estatus. De esta manera, se convierte en la única persona que rompe el prejuicio lingüístico de categorizar a las lenguas según su potencia económica y demográfica.

Dado que la gran mayoría de los informantes han establecido por iniciativa propia una serie de denominaciones hacia el inglés, inmediatamente a continuación de esas declaraciones se ha asumido su marco de referencia con el fin de averiguar qué criterios siguen para señalar tales calificaciones. Para ello, se les ha preguntado si, a su juicio, existen otras lenguas que actualmente le disputen al inglés el terreno mencionado por los propios informantes.

Cada uno ha formulado un ranking propio de lenguas que pueden competir con el inglés por esa hegemonía internacional. Las listas señaladas son bastante dispares, identificando lenguas como el francés, el castellano, el chino mandarín, el alemán, el hindi o el coreano. Seis personas han ofrecido su clasificación particular de lenguas, de entre las cuales solamente tres han justificado su elección con base en tres criterios: número de hablantes, presencia internacional y peso comercial.

Respecto al número de hablantes, Natalia afirma que "después del inglés está el francés porque hay muchos países de habla francesa, en África sobre todo" y Emma asegura que "el chino mandarín es el que más se habla, luego viene el inglés y luego una lengua india [el hindi], creo". Como se observa, ambas entrevistadas establecen un eje de disputa entre lenguas según la cantidad de hablantes que tienen. De esas aseveraciones se puede inferir que tanto Natalia como Emma interpretan que el gran peso demolingüístico del que goza el inglés está formado por hablantes nativos, es decir, personas cuya primera lengua es el inglés.

No obstante, existe una diferencia cuantitativa importante entre el número de hablantes nativos y el conjunto de estos más los hablantes que declaran tener el inglés como segunda lengua. Si se toma la pauta seguida por las entrevistadas, es decir, ordenar las lenguas según el número de hablantes nativos, ninguna de las dos acierta. Según Ethnologue (2015), el chino mandarín y el castellano superan en número de hablantes nativos al inglés -en millones, el chino mandarín cuenta con 900, el castellano con 472 y el inglés con 339. De priorizar el número total de hablantes, tanto de L1 como de L2, solo Emma se acerca más a la clasificación: en este caso, el chino mandarín ocupa el primer puesto con 1.090 millones, seguido del inglés con 942 millones y del castellano con 570 millones (Ethnologue 2015). Como asegura Crystal (2003, p. 69) en referencia al inglés, "there are more people speaking it as a second language, and many more speaking it as a foreign language".

La batalla de cifras a la que se incorporan numerosas instituciones y autores tienen normalmente como objetivo destacar unas lenguas sobre otras como si ese criterio determinase una condición superior de las mismas. No obstante, entre los obstáculos para obtener resultados fidedignos, Tusón Valls (2010, p. 63) señala la dificultad para acceder a los censos de los hablantes de lenguas como el hindi; la propia consideración de hablante de una lengua, ya sea como hablante nativo, como hablante de L2, o si lo son por elección o por imposición histórica; el condicionamiento que implica el contexto y las respuestas políticamente correctas de las personas, entre otros.

Lo interesante no son tanto las cifras sino la ideología lingüística tan poderosa que opera en las informantes y que establece que el inglés cuenta con una cantidad altísima de hablantes que lo sitúa entre las lenguas más habladas del mundo. Esta aseveración se puede matizar dependiendo de varios factores: por un lado, qué se entiende por hablar una lengua, 
pues existen diversos grados de competencia lingüística que son complicados de controlar en las estadísticas; por otro lado, la clasificación varía dependiendo de si se toman únicamente los hablantes de inglés como L1, como L2 o el conjunto de ambos grupos. En todo caso, en las informantes se observa el poder ideológico con el que cuentan aquellas instituciones que promocionan el inglés como lengua internacional y lo hacen ver como un recurso imprescindible en el mundo moderno.

Al hilo de los criterios establecidos por las personas entrevistadas para construir su ranking particular de lenguas, Emma señala a continuación la presencia internacional, ámbito en el que una de las lenguas que destaca no sale bien parada: "tampoco veo que el chino mandarín tenga esa presencia internacional. Desde el punto de vista de la economía, China está creciendo mucho, pero no le veo esa masividad al chino mandarín". Pese a establecer dos criterios a la hora de esa competencia entre lenguas, la informante prioriza finalmente la presencia internacional de las lenguas como requisito primordial para que se disputen el terreno. De esta manera, el factor económico se ensalza como el más importante para hegemonizar el tablero lingüístico. Directamente relacionado con la economía se encuentra el factor del peso comercial, mencionado por Lorena para conferir poderío al coreano.

En definitiva, destaca la iniciativa de la gran mayoría de los entrevistados a la hora de nombrar al inglés con un calificativo que lo construye como una lengua intrínsecamente universal debido a factores de poderío económico. En este sentido, la muestra de informantes ha vislumbrado ese dominio del inglés y, en ciertos casos, ha argumentado los rasgos que le llevan a ser una lengua internacional. Sin embargo, no se perciben reflexiones profundas en cuanto a los orígenes económicos de ese poderío, ratificando así uno de los mayores prejuicios lingüísticos hacia el inglés.

\subsection{Las percepciones en torno a la heterogeneidad del inglés}

Uno de los prejuicios lingüísticos más extendidos en la cultura occidental es el de la existencia de lenguas fijas e inmutables, ajenas a los cambios lingüísticos que deparan el paso del tiempo y la extensión geográfica (Moreno Cabrera, 2004, pp. 50-51). En este caso, se ha querido averiguar el peso de este prejuicio entre las personas entrevistadas. A partir de las respuestas vertidas, se comprueba una unanimidad a la hora de caracterizar el inglés con la heterogeneidad lingüística. Yoel afirma que "el inglés tiene muchas variedades", idea sostenida también por Natalia, quien aporta una experiencia personal: "mi hermano está en EE.UU. y me contó que el inglés de allá es diferente al que él aprendió estando en Cuba, que era el inglés de Inglaterra". Por su parte, Emma destaca una de las causas de esta heterogeneidad, la cual, además, la asocia a todas las lenguas: "a medida que pasa el tiempo, van variando ciertas cosas. No solo el acento, también las palabras. Y no creo que solo le pase al inglés, sino a los idiomas de todos los países". Esta informante asume el paso del tiempo como uno de los motivos por los que las lenguas varían los aspectos prosódicos y léxicos.

Se percibe, incluso, un tono burlesco en Mario cuando ratifica la heterogeneidad del inglés. El informante asume como algo obvio y fuera de discusión el hecho de que esta lengua se hable de manera diferente en distintos lugares. Ese estilo sarcástico revela una posición quínica, en el sentido que señala Sloterdijk (1987, p. 218), puesto que enfrenta el prejuicio lingüístico con un tono trivial e irónico. De esta manera, la muestra de informantes no solo rechaza la supuesta homogeneidad linguiística del inglés, sino que a través de Mario 
se vislumbra una actitud de resistencia ante el prejuicio hegemónico. Para Sloterdijk (1987), esta actitud quiínica es contrarrestada por la cultura dominante con respuestas cínicas. En este sentido, surge una relación dialéctica en la que la cultura dominante niega la negación de Mario para hacer prevalecer el prejuicio lingüístico. Por lo tanto, el resultado de la contraposición no es la síntesis hegeliana (Hegel, 2010, p. 698) en la medida en que no hay movimiento o transformación hacia una conclusión que haya deshecho las proposiciones demostradamente falsas o inservibles. Más bien, la influencia de la que goza este prejuicio prueba que la resistencia quínica de personas como Mario no logra alterar las posiciones en el tablero hegemónico.

Pese a esta unanimidad, llaman la atención tres personas participantes que interpretan las distintas variedades del inglés como adaptaciones de un sistema lingüístico único. Carla afirma que "cada región lo adapta a sus costumbres" y Roberto persiste en esta idea de adaptación alegando que "cada país lo adapta a su pronunciación, a su vocabulario". Como se observa, ambos informantes reconocen la variabilidad del inglés pero la entienden como una adaptación, es decir, como una adecuación de ciertas categorías generales a los rasgos particulares de cada comunidad lingüística. Esas categorías generales pertenecen a una lengua única a la que alude Lorena, la tercera informante mencionada: "cada país tiene su acento, su matiz a la hora de hablar, pero es el mismo inglés. Cada país tiene sus palabras [...] y no deja de ser la misma lengua". La entrevistada explicita lo que se infería de los dos anteriores, y es la idea de la diferencia dentro de la unidad. Los tres participantes reconocen la variabilidad interna del inglés, pero la someten a una categoría jerárquicamente superior, que es la unidad lingüística.

Los tres reflejan una contradicción entre una creencia interna liberada del prejuicio lingüístico -“el inglés es heterogéneo"- y una asunción externa que obedece a un ritual ciego -“el inglés es homogéneo"-, aquello a lo que Bourdieu (1990, p. 10) denominó habitus. Weininger (2005, p. 91) recuerda que no hay que confundir esta noción con la de "hábito", cuyo significado ordinario designa una acción rutinizada y repetitiva. Para ilustrar la conceptualización de habitus, Bourdieu (1990, p. 11) pone el ejemplo de un tenista que corre impulsivamente hacia la pelota sin sopesar las consecuencias esperadas de esa acción. Zupančič (2008, p. 15) recurre a la misma máxima para caracterizar el periodo histórico de la Ilustración, afirmando que la razón se mantuvo impotente en todas las prácticas puesto que, a pesar de ese conocimiento, se siguieron manifestando algunas creencias religiosas.

El habitus en estos tres informantes se manifiesta en tanto que abrazan una ilusión que conscientemente no sostienen, pero la fuerza de posiciones preconscientes inculcadas a través de la experiencia les impone formas ideológicas particulares. La opinión que muestran no se genera asumiendo explícitamente las normas sociolingüísticas ni mediante un cálculo racional de los beneficios y costes de asumir tal idea. Más bien, las personas participantes manifiestan sus opiniones movidos espontáneamente por el habitus, esa ritualización de la práctica social. De esta manera, los parámetros de la acción se encuentran acabados antes de la puesta en práctica de los actores. Las personas entrevistadas no participan en la construcción de lo que sostienen. Frente a la nula interacción, se percibe la interpasividad a la que alude Žižek (2007, p. 23), ya que estas tres personas entrevistadas permanecen al margen de toda elaboración ideológica.

Dado que todas las personas entrevistadas han reconocido la existencia de diferentes variedades del inglés, se ha querido investigar otro de los prejuicios más comunes respecto a la variabilidad lingüística: la existencia de variedades superiores al resto. Este prejuicio se muestra a menudo mediante pares dicotómicos que caracterizan a las lenguas en correctas/incorrectas, 
bonitas/feas o suaves/ásperas, entre otras categorías. En este sentido, una proporción considerable de las personas entrevistadas han vertido este prejuicio lingüístico. Tanto Mario como Alejandro han señalado que en Inglaterra es donde mejor se habla el inglés, mientras que Carla y Lucas no solo apuntan a esta superioridad estructural del inglés británico sino que, además, le otorgan la propiedad de "belleza": "yo creo que donde más bonito se habla es en Inglaterra. A mi criterio, es superior, sî", sentencia Lucas. Estos cuatro informantes reproducen el prejuicio lingüístico de que el inglés británico es cualitativamente superior y más bonito que el resto de variedades de esta lengua.

La primera propiedad asociada por estas personas entrevistadas al inglés británico, la superioridad, se adscribe a la idea de que la norma gramatical estándar es el modelo a seguir por los hablantes, y quienes se desvíen de ella representan una variedad corrupta. Pese a esta idea de que unas lenguas son mejores que otras, Garrido Medina (1997, p. 94) señala que todas las lenguas disponen de procedimientos para crear palabras y construcciones si se da la necesidad de ello en sus comunidades lingüísticas. Los informantes entienden el inglés británico como el estándar, ya que la variedad que se les impartió en la escuela era esta, tal y como Yoel asegura: "aquí desde pequeñitos nos enseñan el inglés de Inglaterra". Este proceso de estandarización, llevado a cabo a partir de la lengua escrita tras el establecimiento de la imprenta como mecanismo global (Burke, 2004, p. 91), genera prejuicios lingüísticos en las personas entrevistadas que les llevan a categorizar las diferentes variedades a través de parámetros externos a lo lingüístico y en relación, más bien, con criterios políticos y económicos.

Para Bourdieu (1985, p. 26), estas falsas impresiones son promovidas por "las instituciones del Estado en general y las academias e instituciones culturales en particular [...] con el fin de privar de valor y marginar el habla espontánea de la mayor parte de la gente común". El habla popular es frecuentemente catalogada como vulgar o impropia, lo que conduce a abrazar prejuicios de este tipo. Diana alude precisamente a la instrucción educativa de la gente como si se tratara de un medio para sortear la incorrección del habla coloquial: "hay variedades más vulgares [...] dependiendo de la cultura de las personas y de dónde sean, de los barrios de los que provengan".

Este prejuicio lingüístico no se encuentra restringido a personas de ámbitos externos a la lingüística, sino que muchos académicos de la lengua han contribuido a su asentamiento. Así, aunque la gramática castellana de Bello es considerada una de las obras más influyentes de la filología española, el autor venezolano sostenía que la incultura y el descuido gramatical de la gente vulgar provocaban una indeseada variabilidad lingüística en la lengua castellana (More 2004, p. 68). No obstante, Moreno Cabrera (2004, p. 154) señala que "lo que ayer era un vulgarismo que ofendía los oídos de las personas refinadas, hoy es un término tan digno de nuestra lengua como el cultismo más refinado". No hay que olvidar que lo que hoy se considera castellano deriva de las supuestas incorrecciones de los antiguos hablantes de la Península Ibérica respecto a la norma culta del latín. De esta manera, algunas de las personas entrevistadas establecen subconscientemente una jerarquía elitista en la que la variedad prestigiosa del inglés solamente puede ser alcanzada por aquellas personas que dominen ciertos capitales socioculturales que, además, les hagan ser percibidos como hablantes legítimos.

La segunda propiedad mencionada por algunos de los entrevistados, la belleza lingüística, se asocia al inglés británico como un atributo endógeno a él. Valoraciones de este tipo muestran un etnocentrismo cultural mediante el que "convertimos en punto absoluto de referencia los patrones fónicos que nos resultan familiares" (Tusón Valls, 2010, p. 56). 
Así, los informantes que categorizan las variedades del inglés según su sonoridad se guían por aparentes condicionantes subjetivos como el gusto por ciertos patrones fonéticos pero que realmente esconden la influencia de una corriente ideológica que reproduce unas características y no otras. Ninguna variedad puede ser intrínsecamente mejor, más bonita o más auténtica que otra, sino que todas ellas satisfacen las necesidades comunicativas de la comunidad que las habla (Bagno, 1999, p. 47).

No es de extrañar el arraigo de estas ideas en el sentir colectivo cuando desde la Antigüedad, numerosos intelectuales las han desarrollado y las han inculcado como si de axiomas se trataran (Tusón Valls, 1994, p. 25). Para Bourdieu (1985, p. 28), esta clase de concepciones que privilegian una variedad por encima del resto "es utilizada como un método poderoso de control social y es una fuente de discriminación y sometimiento". Por lo tanto, aunque buena parte de las personas entrevistadas no reproduce estos prejuicios, la otra parte asume como incuestionables unas ideas que, si bien subconscientemente utilizadas, pueden convertirse en lo que Zuidema (2005, p. 666) denomina “discriminación activa” hacia determinados grupos de hablantes.

\subsection{Apreciaciones sobre el inglés hablado en Cuba}

Además de analizar el prejuicio lingüístico referente a la calidad de diferentes variedades nativas del inglés, se ha querido conocer las opiniones de las personas entrevistadas sobre el inglés hablado por cubanos. Para ello, se les ha pedido identificar algún conocido cubano que, bajo el criterio de las personas participantes, hable bien el inglés. Tras este reconocimiento, han reflexionado en torno a la calidad lingüística de esa variedad frente a la de un hablante nativo estadounidense.

En las respuestas de los informantes se observa un equilibrio entre quienes opinan que ambas variedades tienen la misma calidad y aquellos que superponen el inglés nativo del estadounidense. Los primeros señalan la posibilidad real de que un cubano pueda alcanzar la misma competencia en inglés que un hablante nativo, aunque no ofrecen justificaciones para sostener esta tesis.

No obstante, los participantes que se muestran proclives a asumir la desigualdad entre ambas variedades señalan las diferencias gramaticales y prosódicas como argumentos. Así, Roberto alega que el inglés de la persona cubana "no es de tanta calidad, siempre va a quedar algo, el acento, aunque tenga mucha fluidez", de manera que la perfección consiste en asimilar todos los rasgos lingüísticos de un hablante nativo. Esa perfección también es sostenida por Mario, el cual asegura que la persona cubana que aprende inglés "puede lograr la perfección si vive un tiempo en el país". Estos entrevistados dibujan al inglés como un compartimento lleno de trazos lingüísticos cuyo dominio garantiza la correcta realización de la lengua. En consecuencia, si un hablante cubano desea tener un nivel de inglés equiparable al de un hablante nativo, para este grupo de entrevistados es necesario que domine todos esos rasgos lingüísticos.

Estas personas han reconocido el nivel alto en inglés de sus respectivos conocidos, sentencias acompañadas de cláusulas restrictivas por las que ese conocimiento, pese a ser muy bueno, es inferior al del hablante nativo. El límite que cabría esperar que ejerciese su experiencia personal a la hora de la construcción del prejuicio lingüístico no se realiza. Estas personas reconocen el excelente inglés de sus amigos cubanos que les permite comunicarse 
con hablantes nativos. Aparentemente, esta experiencia podría ejercer una resistencia a la construcción del prejuicio lingüístico en tanto que la experiencia se contrapone a la máxima del prejuicio. Sin embargo, ambos informantes utilizan esa experiencia como un argumento a favor del prejuicio: "el inglés de mi amigo es muy bueno, pero el de un hablante nativo es mejor". Este hecho demuestra que es ineficaz atacar el prejuicio lingüístico diciendo que el inglés del cubano es tan apto como el del nativo. Por el contrario, lo acertado sería manifestar que esa idea sobre el inglés no tiene nada que ver con el inglés.

Lacan (1991, p. 108) ofrece un ejemplo equivalente con el marido patológicamente celoso: aunque realmente su mujer le engañase con otros, lo cual justificaría los fundamentos celosos del marido, dichos celos no dejan de ser una construcción paranoide. Así, aunque el inglés del hablante nativo fuera superior, con la dificultad terminológica que engendra este término, al de una persona cubana, esto no tendría nada que ver con el origen de ese prejuicio lingüístico. Lo adecuado no es, por tanto, declarar que el inglés del hablante nativo no es superior, sino que la idea de superioridad del hablante nativo no tiene nada que ver con la lengua.

A esta altura se plantea una segunda problemática que consiste en interpretar qué entiende este grupo de entrevistados por un dominio semejante al de un hablante nativo. Si, como ya han reconocido a lo largo de la entrevista, existe una multiplicidad de variantes del inglés, a cada una de ellas le corresponde unos trazos lingüísticos que difieren entre sí. Por consiguiente, el dominio de ciertos rasgos puede ser relevante en algunas variedades pero no en todas. Bien es cierto que la pregunta formulada acota el cerco de variabilidad, ya que se ha tomado a un hipotético hablante nativo estadounidense para comparar su inglés con el de una persona cubana. Dejando a un lado las diferencias lingüísticas internas que entraña un país de tal extensión como EE.UU., estos informantes reflexionan con base en un hablante ideal.

Este concepto deriva de la teoría acerca de la competencia comunicativa de Chomsky (1965, p. 3), quien establece que "an ideal speaker-listener, in a completely homogeneous speech-community, [...] knows its language perfectly and is unaffected by such grammatically irrelevant conditions as memory limitation". Este grupo de participantes toma al hablante nativo estadounidense como un hablante ideal que es competente en todas las categorías lingüísticas que marca la norma estándar, las cuales compara con esa persona cubana que aprende el inglés como L2. Mientras que Mario sí concibe la posibilidad de que el entorno sociolingüístico proporcione al hablante cubano todas las herramientas necesarias para ser igual de competente en inglés que un nativo, el resto de los informantes de este grupo concluyen que no se puede alcanzar el nivel de este último.

Este prejuicio lingüístico se basa en considerar que todos los hablantes de una lengua son competentes en esta, lo que les llevaría a poder distinguir las oraciones gramaticales de las oraciones no gramaticales. Sin embargo, este presupuesto no se puede aceptar en tanto que no todos los hablantes nativos de una lengua son capaces de hacer estas distinciones y algunos hablantes no nativos lo pueden hacer incluso con mayor precisión (Cenoz Iragui 2004, p. 450). Por lo tanto, este conjunto de personas entrevistadas defiende una conclusión basada en una premisa falaz que idealiza el concepto de hablante nativo, alejándolo de las relaciones sociolingüísticas que imperan en cualquier sociedad. A este respecto, Vendryes (1921, p. 420) afirma que las lenguas no se pueden tomar como entidades independientes de sus hablantes. Así pues, la hipótesis que maneja este grupo de entrevistados carece de fuerza en tanto que no se puede comparar una variedad del inglés con una supuesta lengua ideal. 


\section{Conclusiones}

A lo largo de esta investigación se han detectado y explicado los prejuicios lingüísticos que presentan las personas participantes en esta investigación. Cabe subrayar que esta muestra de informantes no pretende representar a la juventud cubana en su totalidad. Más bien, este trabajo cualitativo pretende señalar una serie de ideologías lingüísticas concretas en torno al inglés a partir de las cuales otros análisis de carácter cuantitativo y cualitativo pueden completar para contribuir al conocimiento de este tema específico. Se detecta una proporción considerable de personas participantes que ratifican prejuicios muy comunes como la supuesta internacionalidad del inglés, la homogeneidad estructural de esta lengua o la superioridad de unas variedades lingüísticas sobe otras.

En primer lugar, la mayoría de las personas entrevistadas asumen la internacionalidad o universalidad como propiedades consustanciales al inglés. Las causas de esta característica son diversas: el gran número de hablantes que posee el inglés, la presencia internacional o el peso comercial. La primera razón se ha demostrado poco consistente dadas las dificultades metodológicas a la hora de acceder a censos fiables del número de hablantes, así como la controversia de definir al hablante nativo. La segunda y la tercera razón vertidas aluden directamente a un factor económico que se convierte en un criterio crucial para las personas entrevistadas cuando se trata de juzgar las lenguas.

Así pues, se corrobora la primera hipótesis de la que parte esta investigación, aunque no se esperaba una caracterización tan manifiesta sino que se pensaba que habría que inferir ese prejuicio a través de las opiniones y experiencias que contasen los informantes. Por su parte, la segunda hipótesis queda parcialmente ratificada en la medida en que solo una parte de las personas entrevistadas han dado señas de asumir el inglés como lengua franca.

En segundo lugar, se detecta un rechazo unánime al prejuicio lingüístico que construye al inglés como una lengua uniforme y homogénea. La totalidad de la muestra reconoce la variabilidad que presenta esta lengua, manifestada en rasgos gramaticales y prosódicos. Pese a este veredicto general, es posible detectar en varios informantes una ideología subrepticia que consiste en ver la variedad dentro de la uniformidad. Así, estas personas construyen el inglés como una herramienta con una serie de normas y rasgos compartidos que posteriormente son adaptados por los hablantes, los cuales modifican ciertas características lingüísticas para acoplarlas a su idiosincrasia. Por lo tanto, destaca el consenso en torno a la heterogeneidad del inglés, aunque ciertas personas entrevistadas la subordinan a una norma común.

En tercer lugar, en la muestra de informantes se confirma un prejuicio lingüístico muy poderoso que confiere a las lenguas ciertas cualidades como si de propiedades intrínsecas se tratasen, aunque bien es cierto que existen excepciones de personas entrevistadas que no muestran tales prejuicios. Así, la variedad del inglés de Inglaterra se privilegia por encima de otras variedades en torno a dos ejes: el de lengua correcta/incorrecta y el de lengua bonita/fea. Contrariamente a lo que se pensaba en las hipótesis, la variedad considerada superior por una proporción considerable de las personas entrevistadas es el inglés británico, no estadounidense. Esta opinión demuestra que varios informantes ven en las variedades lingüísticas trazos de superioridad e inferioridad entre ellas.

Tales categorías, además, resaltan cuando se trata de comparar las variedades del inglés de un hablante nativo y de una persona cubana que la aprende como L2. La mitad de las personas entrevistadas han considerado que el inglés de la persona cubana es de menor calidad 
que el de un hablante nativo. Así, se confirma parcialmente la cuarta hipótesis en la medida en que existe proporción entre quienes la ratifican y quienes no.

En definitiva, a partir de la muestra de participantes que se ha investigado para este trabajo, se puede afirmar que la juventud cubana presenta ciertos prejuicios lingüísticos en relación con el inglés, aunque, cabe señalar que en ciertas ocasiones hay excepciones a la opinión mayoritaria y, en otras, esas opiniones se muestran como ideologías subconscientes contrapuestas a las ideas conscientes de las propias personas entrevistadas.

\section{Bibliografía}

Ackerman, N. W. y Jahoda, M. (1950). Anti-Semitism and Emotional Disorder. Nueva York: Harper.

Alfaraz, G. (2002). Miami Cuban perceptions of varieties of Spanish. En D. Long y D. R. Preston (Eds.). Handbook of perceptual dialectology, II. (pp. 1-11). Ámsterdam/ Filadelfia: John Benjamins Publishing Company.

Allport, G. W. (1962). La naturaleza del prejuicio. R. Malfé (trad.). Buenos Aires: Editorial Universitaria de Buenos Aires.

Bagno, M. (1999). Preconceito lingüístico. O que é, como se faz. São Paulo: Loyola.

Bourdieu, P. (1985). ¿Qué significa hablar? Economía de los intercambios lingüísticos. E. Martínez Pérez (trad.). Madrid: Akal.

Bourdieu, P. (1990). In Other Words: Essays towards a Reflexive Sociology. M. Adamson (trad.). Stanford, CA: Stanford University Press.

Bourdieu, P. (1997). Méditations pascaliennes. París: Seuil.

Burke, P. (2004). Languages and Communities in Early Modern Europe. Cambridge: Cambridge University Press.

Canals Fleitas, W. (1999). Marginalidad: Un acercamiento al tema a partir de las actitudes lingüísticas manifiestas por el hablante desde los lustros coloniales hasta la actualidad. (Tesis de Licenciatura). Universidad de La Habana.

Cenoz Iragui, J. (2004). El concepto de competencia comunicativa. En J. Sánchez Lobato e I. Santos Gargallo (Eds.). Vademécum para la formación de profesores. Enseñar español como segunda lengua/lengua extranjera. (pp. 449-465). Madrid: SGEL.

Chomsky, N. (1965). Aspects of the Theory of Syntax. Massachusetts: The MIT Press.

Crystal, D. (2003). English as a global language. Cambridge: Cambridge University Press.

Del Rincón Igea, D. (1995). Técnicas de Investigación en Ciencias Sociales. Madrid: Dykinson.

Dohotaru, P. (1998-1999). Condicionamiento lingüístico y social de la variación de -/R/ en el habla de habaneros universitarios. Anuario de Literatura y Lingüística, 29 (30), 51-76.

Dohotaru, P. (2000). Variación sociolingüística de /s/, /R/ y /l/ en el habla de universitarios habaneros. (Tesis doctoral). Instituto de Literatura y Lingüística de La Habana.

Dohotaru, P. (2002). La variación de -/l/ en el habla espontánea de habaneros universitarios. En M. Aleza Izquierdo y N. Gregori Torada (Coords.). Estudios lingüísticos Cubanos. II: Homenaje a Leandro Caballero Díaz. (pp. 67-86). Valencia: Universitat de València. 
Dörnyei, Z. (2007). Research Methods in Applied Linguistics. Quantitative, Qualitative and Mixed Methodologies. Oxford: Oxford University Press.

Edwards, J. (1994). Multilingualism. Londres/Nueva York: Routledge.

Ethnologue (2015). Summary by language size. Recuperado de https://www.ethnologue.com/ statistics/size.

Fuster, N. N. (2012). Percepciones y actitudes lingüísticas de habaneros y santiagueros en Cuba. (Tesis de Maestría). Universidad de Puerto Rico.

Garrido Medina, J. (1997). Estilo y texto en la lengua. Madrid: Gredos.

Gregori Torada, N. (1993-1994). Identidad, prestigio y estigmatización lingüísticas en el Caribe Hispánico. Anuario de Literatura y Lingüística, 24 (25), 28-39.

Hegel, G. W. F. (2010). The Science of Logic. G. di Giovanni (trad.). Cambridge: Cambridge University Press.

House, J. (2003). English as a lingua franca: A threat to multilingualism?. Journal of Sociolinguistics, 7 (4), 556-578.

Iglesias, A. (2012). Actitudes e prexuízos lingüísticos en Galicia. A súa influencia nos usos. A letra miúda, 1, 1-18.

Kroskrity, P. V. (2010). Language ideologies: Envolving perspectives. En J. Jaspers, J. Östmam y J. Verschueren (Eds.). Society and Language Use. (pp. 192-211). Ámsterdam/ Filadelfia: John Benjamins Publishing Company.

Kvale, S. (2006). Dominance through interviews and dialogues. Qualitative Inquiry, 12 (3), 480-500.

Lacan, J. (1991). Le Séminaire, livre XVII: L'envers de la psychanalyse. París: Seuil.

More, B. (2004). La construcción ideológica de una base empírica: selección y elaboración en la gramática de Andrés Bello. En J. del Valle y L. G. Stheeman (Eds.). La batalla del idioma: la intelectualidad hispánica ante la lengua. (pp. 67-92). Madrid: Iberoamericana.

Moreno Cabrera, J. C. (2004). La dignidad e igualdad de las lenguas. Crítica de la discriminación lingüística. Madrid: Alianza.

Pennycook, A. (1994). The Cultural Politics of English as an International Language. Londres: Longman.

Pinto Pajares, D. (2016). Ideologías y actitudes lingüísticas de la juventud cubana hacia el inglés. Diálogo de la lengua, 8, 97-117.

Sapir, E. (1921). Language: An Introduction to the Study of Speech. Nueva York: Harcourt, Brace.

Sloterdijk, P. (1987). Critique of Cynical Reason. M. Eldred y A. Huyssen (trads.). Minneapolis, MN: Minnesota University Press. 
Sobrino Triana, R.; Montero Bernal, L. E. y Menéndez Pryce, A. J. (2014). Actitudes lingüísticas en Cuba. Cambios positivos hacia la variante nacional de la lengua. En A. B. Chiquito y M. A. Quesada Pacheco (Eds.). Actitudes lingüísticas de los hispanohablantes hacia el idioma español y sus variantes, 5. (pp 290-408). Bergen: Language and Linguistic Studies.

Spinoza, B. (2000). Ética demostrada según el orden geométrico. (A. Domínguez, trad.). Madrid: Trotta.

Tusón Valls, J. (1994). Prejuicios lingüísticos y enseñanza. Teoría y práctica de la educación, $11,22-27$.

Tusón Valls, J. (2010). Los prejuicios lingüísticos. Barcelona: Octaedro.

Van Dijk, Teun. (1998). Ideology. A Multidisciplinary Approach. Londres/Thousand Oaks/ Nueva Delhi: SAGE.

Velázquez Pratts, H. (2009). Las actitudes lingüísticas en Holguín. Ilustrados. [Versión digital]. Recuperado de: http://www.ilustrados.com/tema/5276/actitudes-linguisticas-Holguin.html.

Vendryes, J. (1921). Le langage. Introduction linguistique à l'histoire. París: Renaissance du livre.

Watson, M. S. (2006). Post-revolutionary Cuban Spanish: Changes in the lexicon and language attitudes motivated by socio-political reforms. (Tesis doctoral). Georgetown, WA: Georgetown University.

Weininger, E. B. (2005). Foundations of Pierre Bourdieu's class analysis. En E. Olin Wright (Ed.). Approaches to Class Analysis. (pp. 82-118). Cambridge: Cambridge University Press.

Žižek, S. (2007). How to Read Lacan. Nueva York: W. W. Norton \& Company.

Zuidema, L. A. (2005). Myth education: Rationale and strategies for teaching against linguistic prejudice. Journal of Adolescent \& Adult Literacy, 48 (8), 666-675.

Zupančič, A. (2008). The Odd one in: On Comedy. Cambridge: MIT Press. 
